\section{PD-1 blockade as bridge to allogeneic stem cell transplantation in relapsed/refractory Hodgkin lymphoma patients: a retrospective single center case series}

Classical Hodgkin lymphoma (cHL) patients who fail autologous stem cell transplantation (ASCT) have extremely poor prognosis with a median overall survival (OS) of two years. ${ }^{1}$ In this setting, treatment with brentuximab vedotin (BV) resulted in an objective response rate (ORR) of $75 \%$, with $35 \%$ complete response (CR). Nevertheless, most patients required additional treatment within one year ${ }^{2}$ and still represent an unmet clinical need.

Recent studies suggest that malignant Hodgkin ReedSternberg cells can evade host immune surveillance by increasing the expression on their surface of programmed death 1 ligands (PD-L1 and PD-L2) due to a copy number alteration involving chromosome 9p24.1.3 The amplification of 9p24.1 may also include JAK2, increasing activity of the JAK-STAT pathway that further induces PD-1 ligand transcription. ${ }^{3}$ Physiologically, the interaction between PD1 and its ligands limits T-cell mediated immune responses, making cytotoxic $\mathrm{T}$ lymphocytes temporarily ineffective. Therefore, increased PD-L1 and PD-L2 expression by Reed-Sternberg cells contributes to an ineffective immune-cell microenvironment of cHL, leading to escape from the host immune surveillance and the tumor growth. ${ }^{4}$ This unique dependence on the PD-1 pathway allowed a rational use of anti PD-1 monoclonal antibodies (namely nivolumab and pembrolizumab) to treat patients with cHL. PD-1 blockade resulted in high ORR (approx. $70 \%$ ) with an acceptable safety profile, allowing recent US Food and Drug Administration (FDA) and European Medicines Agency (EMA) approval of nivolumab and pembrolizumab for the treatment of adult patients with $\mathrm{cHL}$ who have relapsed or progressed after ASCT and BV or at least three systemic therapies including $\mathrm{BV}$.

Long-term survival results are lacking, nor do we know which kind of patients will eventually achieve a durable remission or who can benefit from a consolidation with stem cell transplantation (SCT).

Although allogeneic SCT (alloSCT) is still a curative treatment option for those patients with highly chemorefractory disease (especially for those who are relapsed after/refractory to alloSCT), ${ }^{7}$ the safety and efficacy of SCT seems to be different in patients previously exposed to PD-1 inhibitors. In fact, their immune-mediated mechanism results in a prolonged clinical activity and in a long-lasting disturbance in the composition of the circulating T-cell population. ${ }^{8}$ Specifically, residual PD-1 inhibition can enhance donor cytotoxic T-lymphocyte (CTL) response, which translates into two opposite effects: (i) an augmented graft-versus-lymphoma (GvL) effect, due to a restored CTL sensitivity against residual ReedSternberg cells; ${ }^{9}$ and (ii) an increasing incidence or severity of acute graft-versus-host disease (aGvHD) and of other immune complications, mainly due to a loss of donor-CTL regulation mediated by PD-L1, expressed on host antigen presenting cells. Overall, recently published data show that alloSCT after PD-1 blockade seems to have a more favorable outcome than historical series, even if it increases early toxicity (aGvHD and immune toxicity), suggesting that the PD-1 blockade should not preclude alloSCT. ${ }^{5,8,10}$

We report our experience in the treatment of relapsed/refractory $(\mathrm{R} / \mathrm{R}) \mathrm{cHL}$ patients with anti-PD-1
$\mathrm{mAb}$ used as bridge to alloSCT, to assess the effectiveness and the safety of this therapeutic approach outside a clinical trial context.

This retrospective study was approved by our institutional board. All participants gave written informed consent in accordance with the Declaration of Helsinki.

The primary end point of the study was ORR; secondary end points were OS and PFS calculated from initiation of anti-PD1 therapy, and the safety profile.

From February 2014 to November 2016, 13 patients affected by R/R cHL were treated with an anti PD- $1 \mathrm{mAb}$ ( 3 with pembrolizumab and 10 with nivolumab) prior to alloSCT. Median age at the time of diagnosis was 33.5 years (range: 16.0-51.6 years). Patients received a median of five previous systemic treatments (range: 4-10), 10 out of 13 were refractory to the first line of chemotherapy (ABVD) and all had previously failed ASCT. Ten patients relapsed after and three were refractory to alloSCT. Twelve subjects were treated with BV (in 5 cases prior to alloSCT): four patients obtained an objective response [2 $\mathrm{CR}$ and 2 partial response (PR)] and then relapsed, while eight were refractory to BV.

Patients received a median of 16 cycles (range: 6-29 cycles) of anti-PD-1 mAb. The ORR to PD-1 therapy was $84.6 \%$ : 4 of 13 patients (30.8\%) achieved a CR and seven a PR. Two patients had an immune-related adverse event (AE) related to $\mathrm{PD}-1$ inhibitor treatment. One subject (patient 1) had a grade 2 hypothyroidism and the other (patient 7) had a grade 4 hepatitis, resolved with pembrolizumab discontinuation and corticosteroids. Ten patients suspended the treatment due to the achievement of an objective response, two for a progression disease (PD); and one for extra-hematologic toxicity (hepatitis and cytomegalovirus pneumonia, patient 7).

The median time from the last dose of PD-1 inhibitor to alloSCT was 51 days (range, 26-98 days). At alloSCT, four patients were in $\mathrm{CR}$, seven in $\mathrm{PR}$, and the remaining two were transplanted with persistent disease. Eleven patients received peripheral blood stem cells as a graft source: seven from unrelated donors [6 matched unrelated donors (MUD); and 1 mismatched (MMUD)] and four from haploidentical donors. The other two patients received bone marrow cells: one from MMUD and one from haploidentical donor.

All patients but one had a reduced-intensity conditioning regimen to alloSCT and received a T-cell depleted graft; in particular, in eight patients, ATG was added to the conditioning regimen, leading to in vivo T-cell depletion (see Online Supplementary Table S1 for details).

All patients achieved a CR with alloSCT (4 consolidated the previous CR while 7 moved from a PR to a CR and 2 from a PD to a CR) leading to a CR rate of $100 \%$. At the last available follow up, ten patients still show a response (range: $12-47$ months) with a median follow up of 34.3 months. Three patients $(23 \%)$ relapsed after 3,13 and 14 months, respectively: two of them (patients 2 and 12) were in PR and one (patient 8) was in PD before alloSCT. All of them had a MUD, two received a reduced conditioning regimen with ATG-F (patients 2 and 12), the other (patient 8 ) had a myeloablative regimen without ATG. Patient 2 decided not to undergo further therapies. Patient 8 was re-treated with bendamustine (PR) and received donor lymphocyte infusions but then died eight months later due to grade III/IV hepatic aGvHD. Patient 12 started pembrolizumab and achieved a PR; a search for a new unrelated donor is ongoing.

Progression-free and OS were $75.5 \%$ and $90.9 \%$ at 57.4 months, respectively. To date, no patients have died from PD. 
All patients had complete donor chimerism at day 100 and nobody experienced a graft rejection. Five out of 13 patients $(38.5 \%)$ developed an aGvHD, with a median day of onset of 30 days (range: $+21 /+45$ days). These five patients only had skin involvement: one grade 2-3 and the others grade 1-2. The patient with highest grade of aGvDH was the one who developed grade 2 hypothyroidism due to PD-1 blockade therapy (patient 1). Three patients developed a chronic GvHD (cGVHD): one in the skin (grade 3-4), one in the skin, eyes and liver (all grade 2 ), and one in the skin, liver (grade 2) and bowel (grade 3 ). Among the patients who experienced a cGvHD, two are in continuous CR while one has relapsed (patient 2) 14 months after alloSCT. There was only one treatedrelated death due to a grade III-IV hepatic aGvHD (patient 8).

Fifty-four percent of patients (7 of 13) had a non-infectious fever. All patients were started on corticosteroids $(1 \mathrm{mg} / \mathrm{Kg})$ within two weeks of fever onset, with rapid benefit.

The recent FDA and EMA approvals of nivolumab and pembrolizumab for the treatment of adult patients with cHL who have relapsed or progressed after alloSCT and $\mathrm{BV}$ has given rise to many questions about the current role of alloSCT in R/R HL and its efficacy and safety in patients previously exposed to PD-1 inhibitors. To date, the few clinical data available, coming from small heterogeneous cohorts of patients treated with anti-PD1 mAb at any point prior to SCT, suggest that checkpoint blockade therapy before alloSCT has a favorable overall outcome, even if it may increase early toxicity, such as aGvHD and non-infectious febrile syndrome. ${ }^{8,10}$ In the largest series available, among the 31 patients with cHL who underwent to alloSCT after prior PD-1 blockade, the 1 -year cumulative incidence of relapse was $10 \%$. However, a higher than expected rate of early severe transplant-related complications was observed.

We show that alloSCT after PD1 blockade may be associated with promising survival outcome and low relapse rate. A CR rate of $100 \%$ after transplantation was observed and, with a median follow up of 34.3 months, only three patients have relapsed. The overall incidence of acute and chronic GvHD is similar to that previously observed $;{ }^{5}$ in particular, $38.5 \%$ of patients (5 of 13) experienced an aGvHD (only 1 patient a grade II-III). All of them recovered from graft disease quickly with no correlation between the incidence of graft and stem cell source. Seven patients (54\%) experienced a non-infectious febrile syndrome, which resolved with prolonged steroid treatment.

Hodgkin lymphoma patients previously treated with
PD1 inhibitors should not be excluded a priori from SCT, but we do point out that there is a high risk of transplantrelated toxicities and acute immune-related AE can occur.

Beatrice Casadei, Alessandro Broccoli, Vittorio Stefoni, Cinzia Pellegrini, Miriam Marangon, Alice Morigi, Laura Nanni, Ginevra Lolli, Matteo Carella, Lisa Argnani, Michele Cavo and Pier Luigi Zinzani.

Institute of Hematology "L. e A. Seràgnoli", University of Bologna,

Bologna, Italy

Correspondence: PIER LUIGI ZINZANI.

pierluigizinzani@unibo.it.

doi:10.3324/haematol.2019.215962

Information on authorship, contributions, and financial \& other disclosures was provided by the authors and is available with the online version of this article at www. haematologica.org.

\section{References}

1. Arai S, Fanale M, DeVos S, et al. Defining a Hodgkin lymphoma population for novel therapeutics after relapse from autologous hematopoietic cell transplant. Leuk Lymphoma. 2013;54(11):25312533.

2. Chen R, Gopal AK, Smith SE, et al. Five-year survival and durability results of brentuximab vedotin in patients with relapsed or refractory Hodgkin lymphoma. Blood. 2016;128(12):1562-1566.

3. Green MR, Monti S, Rodig SJ, et al. Integrative analysis reveals selective 9p24.1 amplification, increased PD-1 ligand expression, and further induction via JAK2 in nodular sclerosing Hodgkin lymphoma and primary mediastinal large B-cell lymphoma. Blood. 2010;116(17):3268-3277

4. Yamamoto R, Nishikori M, Kitawaki T, et al. PD-1-PD-1 ligand interaction contributes to immunosuppressive microenvironment of Hodgkin lymphoma. Blood. 2008;111(6):3220-3224

5. Armand P, Engert A, Younes A, et al. Nivolumab for Relapsed/Refractory Classic Hodgkin Lymphoma After Failure of Autologous Hematopoietic Cell Transplantation: Extended FollowUp of the Multicohort Single-Arm Phase II CheckMate 205 Trial. J Clin Oncol. 2018;36(14):1428-1439.

6. Chen R, Zinzani PL, Fanale MA, et al. Phase II Study of the Efficacy and Safety of Pembrolizumab for Relapsed/Refractory Classic Hodgkin Lymphoma. J Clin Oncol. 2017;35(19):2125-2132.

7. Rashidi A, Ebadi M, Cashen AF. Allogeneic hematopoietic stem cell transplantation in Hodgkin lymphoma: a systematic review and meta-analysis. Bone Marrow Transplant. 2016;51(4):521-528.

8. Merryman RW, Kim HT, Zinzani PL, et al. Safety and efficacy of allogeneic hematopoietic stem cell transplant after PD-1 blockade in relapsed/refractory lymphoma. Blood. 2017;129(10):1380-1388.

9. Michonneau D, Sagoo P, Breart B, Garcia Z, Celli S, Bousso P. The PD-1 Axis Enforces an Anatomical Segregation of CTL Activity that Creates Tumor Niches after Allogeneic Hematopoietic Stem Cell Transplantation. Immunity. 2016;44(1):143-154.

10. Herbaux C, Merryman R, Devine S, et al. Recommendations for managing PD-1 blockade in the context of allogeneic HCT in Hodgkin lymphoma: taming a necessary evil. Blood. 2018;132(1):916. 